\title{
Deep Learning in Manufacturing
}

\author{
Matthew N. O. Sadiku, Guddi K. Suman, and Sarhan M. Musa \\ Roy G. Perry College of Engineering \\ Prairie View A\&M University \\ Prairie View, TX 77446 \\ U.S.A
}

\begin{abstract}
Deep learning is presently receiving a lot of attention. It is a subset of machine learning, based on multi-layer neural networks or deep neural networks. It is a novel, data-hungry, and high-accuracy analytics approach. This paper discusses deep learning algorithms and their applications in manufacturing.
\end{abstract}

Key Words: Deep Learning, Deep Machine Learning, Hierarchical Learning, Manufacturing.

\section{INTRODUCTION}

One of the most vital industries in the world is manufacturing. The heart of any manufacturing system is the movement of raw materials, goods, and parts. The search for high efficiency and enhanced productivity have always been at the heart of manufacturing. In today's manufacturing, technology is the determining factor for securing competitive advantage. Innovative ideas in the field of artificial intelligence in general and deep machine learning in particular can significantly improve the quality control tasks in a large assembly line. These technologies have come to rescue organizations such manufacturing drowned in the deluge of data, data explosion, and information overload. In manufacturing, they are business critical.

Artificial intelligence (AI) has seen a recent surge in use across a wide variety of industries. One type of AI currently being used in image processing is called deep learning (DL). As illustrated in Figure 1, deep learning is a subset of machine learning which, in turn, is a subset of artificial intelligence [1]. DL is a new area emerging from machine learning and multilayer neural network. It refers to a family of approaches that have taken machine learning to a new level, helping computers make sense out of vast amounts of data in the form of text, images, and sound. It has come to the forefront as a way of overcoming the limitations of neural networks when they are employed to real-world challenges [2].

\section{OVERVIEW OF DEEP LEANRING}

Machine learning refers to algorithms that learn from data. The learning process of these algorithms can either be supervised or unsupervised, depending on the data being used to feed the algorithms. Deep learning algorithms can be regarded as an evolution of machine learning algorithms. Thus, deep learning (DL), or also known as deep neural networks (DNN), refers to a set of machine learning techniques that utilize neural networks with many hidden layers for tasks, where the goal of each layer is to learn to transform input data into a non-linear and more abstract representation. It has become an analytical tool that has attracted more and more attention from researchers in different areas of research in recent years.

The term "deep learning” was coined in 2006 by Geoffrey Hinton and Ruslan Salakhutdinov [3]. Deep learning has given a new dimension to the machine learning research. Deep learning algorithms use neural networking to process massive data sets in order to execute a specified task. They have been used to predict the quality attribute (output) with the process parameters (input). Data is the key in deep learning's effectiveness. Deep-learning techniques can automatically learn complex high-level data features from a large amount of data. Traditionally, a deep learning model has to be retrained every time a new rule is included.

Deep learning relies on a layered structure of algorithms called an artificial neural network (ANN), which is inspired by the functionality of our brain cells called neurons. Consider the example ANN in the Figure 2 [4]. The leftmost layer is called the 
input layer, the rightmost layer is the output layer. Each layer contains one or more neurons. These multilevel layers allow models to detect and train from their own mistakes. The term "deep" in deep learning refers to the number of layers in ANN.

The method that brought significant success to DL is called the Convolutional Neural Network (CNN),proposed by Yann LeCun et al. in 1998. CNN is poised to take over from human operators to spot and detect visual clues.

Advancing DL systems often involves three kinds of engineers: (1) data scientists with domain experts in medical, economic, or climate; (2) hardware designers that develop specialized hardware; and (3) performance and compiler engineers that optimize software [5].

DL is powerful in discovering complex structures in high-dimensional data. It is widely used in many applications such as computer vision, natural language processing, and big data analysis. In addition, DL has several applications in the manufacturing domain. Examples of deep learning in manufacturing include sales forecasting and advanced analytics. Deep Learning is bringing benefits to the design phase, evaluation, production, operation control, maintenance, and sustainment.

\section{APPLICATIONS}

Many fields of research have used deep learning tools to facilitate the processing of massive data. Applications of deep learning are almost limitless, with different applications employing different deep learning models. Deep learning has matured from being a special purpose machine learning technique to a general purpose machine learning tool. It has enjoyed success in various applications such as automatic speech recognition, image recognition, computer vision, object detection, bioinformatics, drug discovery and information retrieval, industrial machines, and manufacturing [6]. In manufacturing, deep learning has the following applications.

- Smart manufacturing: This refers to using advanced data analytics to complement physical science for improving system performance and decision making. It is a fully integrated collaborative system that uses sensors, Internetconnected machines, and big data to monitor the production process and to improve manufacturing efficiencies. Smart manufacturing envisions systems that provide us with insightful information about every step of a product lifecycle. Information-system-enabled smart manufacturing has increased productivity and quality of industrial organizations. Smart manufacturing will enrich the lives of consumers by providing goods and services with high quality and at an affordable cost. Deep learning is applied in the predictive analytics for defect prognosis, i.e. maintenance and service prediction. Deep learning provides advanced analytics tools for processing and analyzing big data in manufacturing and make manufacturing "smart," as shown in Figure 3 [7].

- Automotive industry: The automotive industry covers a wide range of vehicles. Deep learning has many potential applications in the automotive industry during development, manufacturing, and sales. It is also useful in advanced driving assistance systems, autonomous driving, and advanced detection controls [8].

- Predictive Maintenance: In predictive maintenance, data is collected over time to monitor and find patterns to predict failures. Although predictive maintenance is applied in many industries, it thrives in manufacturing. AI in manufacturing has come a long way with technologies like predictive maintenance. The adoption of machine learning and deep learning in manufacturing will only improve predictive maintenance. Deep learning can aid in the predictive maintenance of complex machinery and connected systems.

- Automation: In the hope of decreasing costs and increasing quality and the push for higher volumes of output with lower investments, manufacturing industry is making efforts to achieve a higher degree of automation. For years, machine builders in the automation field of assembly equipment have been focusing on the mechanics of machines.

- Robotics: Deep learning architectures enable robots to learn on their own. Companies use industrial robots to handle complicated and dangerous processes. A robot can train itself for new tasks by object and pattern recognition capabilities of deep learning models. Deep learning models have proven to be compelling in areas such as financial aspects, timesetting data management and money related. 
- Additive manufacturing (AM): This is a new paradigm in design-driven build of customized products. Machine learning and deep learning can be adopted for both analysis and design of microlattices, which can be fabricated using additive manufacturing techniques [9]. Figure 4 shows an example of additive manufacturing [10].

Other applications of deep learning in manufacturing include machining process, process monitoring, continuous manufacturing, pharmaceutical manufacturing, and medical device manufacturing.

\section{BENEFITS}

Deep learning techniques are emerging approaches from machine learning. Manufacturing organizations that adopt DL models derive knowledge, understanding, insight, and foresight to improve their products, processes, and decisions, thereby giving them a competitive advantage. Other benefits include:

- Deep learning techniques are achieving unprecedented levels of accuracy. It shows trends and behaviors that influence the direction of technology.

- They advance current manufacturing process by efficiently handling big data in a rapid pace and with autonomy.

- Deep learning algorithms can outperform humans at recognizing and classifying images.

- Deep learning requires little human intervention to function properly.

- Deep learning can integrate seamlessly with the ambitious goals of Industry 4.0.

\section{CHALLENGES}

A major challenge facing deep learning in various applications include:

- Deep learning requires vast amounts of data. There is the need to store and process large volumes of data as well as dealing with unstructured data.

- $\quad$ Deep learning requires substantial computing power.

Despite that deep learning is emerging as a very powerful technique, it is attracting some criticism. Techniques based on deep learning can learn complex.

\section{CONCLUSION}

Deep learning may be regarded as a software technology designed by programmers to teach computers to do what humans have been doing: learning by example or learning from data. Deep learning has attracted attention in several fields of research because it allows the extraction of features from processing large data. Leading tech companies by the likes of Google, Microsoft, and Apple have invested heavily in DL.

Deep learning is all around us in the form of Siri, Alexa, and Google Assistant. Although deep learning is still in its infancy in many fields of study, its breakthroughs are driving the AI boom and providing a bright future for AI. More information about deep learning in manufacturing can be found in the books in [11,12] and related journal:

Journal of Manufacturing Systems

\section{REFERENCES}

[1] A. Wolfewicz, "Deep learning vs. machine learning - What's the difference?" May 2021, https://levity.ai/blog/differencemachine-learning-deep-learning

[2] M. N. O. Sadiku, M. Tembely, and S. M. Musa, ”Deep learning,” International Research Journal of Advanced Engineering and Science, vol. 2, no. 1, 2017, pp. 77,78.

[3] G. E. Hinton and R. R. Salakhutdinov, "Reducing the dimensionality of data with neural networks," Science, vol. 313, 2006, pp. 504-507.

[4] S. Singh, "Cousins of artificial intelligence," May 2018, https://towardsdatascience.com/cousins-of-artificial-intelligence-dda4edc27b55

[5] A. Rodriguez, "Deep learning systems: Algorithms, compilers, and processors for large-scale production," Synthesis Lectures on Computer Architecture, vol. 15, no. 4, October 2020, pp. 1-265. 
[6] "Deep learning," Wikipedia, https://en.wikipedia.org/wiki/Deep_learning

[7] J. Wang et al., "Deep learning for smart manufacturing: Methods and applications," Journal of Manufacturing Systems, 2018.

[8] A. Luckow et al., "Deep learning in the automotive industry: Applications and tools," Proceedings of 2016 IEEE International Conference on Big Data (Big Data), December 2016.

[9] N. Després et al., "Deep learning and design for additive manufacturing: A framework for microlattice architecture," JOM, vol 72, 2020, pp. 2408-2418.

[10] D. Butcher, “Additive manufacturing in the medical device industry," November 2020, https://www.mastercontrol.com/gxplifeline/additive-manufacturing-in-the-medical-device-industry/

[11] F. Chollet, Deep Learning with Python. Shelter Island, NY: Manning Publications Co., 2018.

[12] E. Stevens, L. Antiga, and T. Viehmann, Deep Learning with PyTorch. Shelter Island, NY: Manning Publications Co., 2020 .

\section{ABOUT THE AUTHORS}

Matthew N.O. Sadiku is a professor emeritus in the Department of Electrical and Computer Engineering at Prairie View A\&M University, Prairie View, Texas. He is the author of several books and papers. His areas of research interest include computational electromagnetics and computer networks. He is a fellow of IEEE.

Guddi K. Suman is currently working towards a PhD in Electrical and Computer Engineering at Prairie View A\&M University, Prairie View, TX. She is working as a Ph.D. intern at Pacific Northwest National Lab in Richland, WA. Her areas of research interest include space radiation effects on electronics, nanomaterials, characterization of nanomaterials and semiconductors, thin film nanofabrication, and nanosensors. She is a student member of IEEE.

Email: guddikarna@gmail.com

Sarhan M. Musa is a professor in the Department of Electrical and Computer Engineering at Prairie View A\&M University, Texas. He has been the director of Prairie View Networking Academy, Texas, since 2004. He is an LTD Sprint and Boeing Welliver Fellow. His areas of research interest include computational electromagnetics and computer networks.

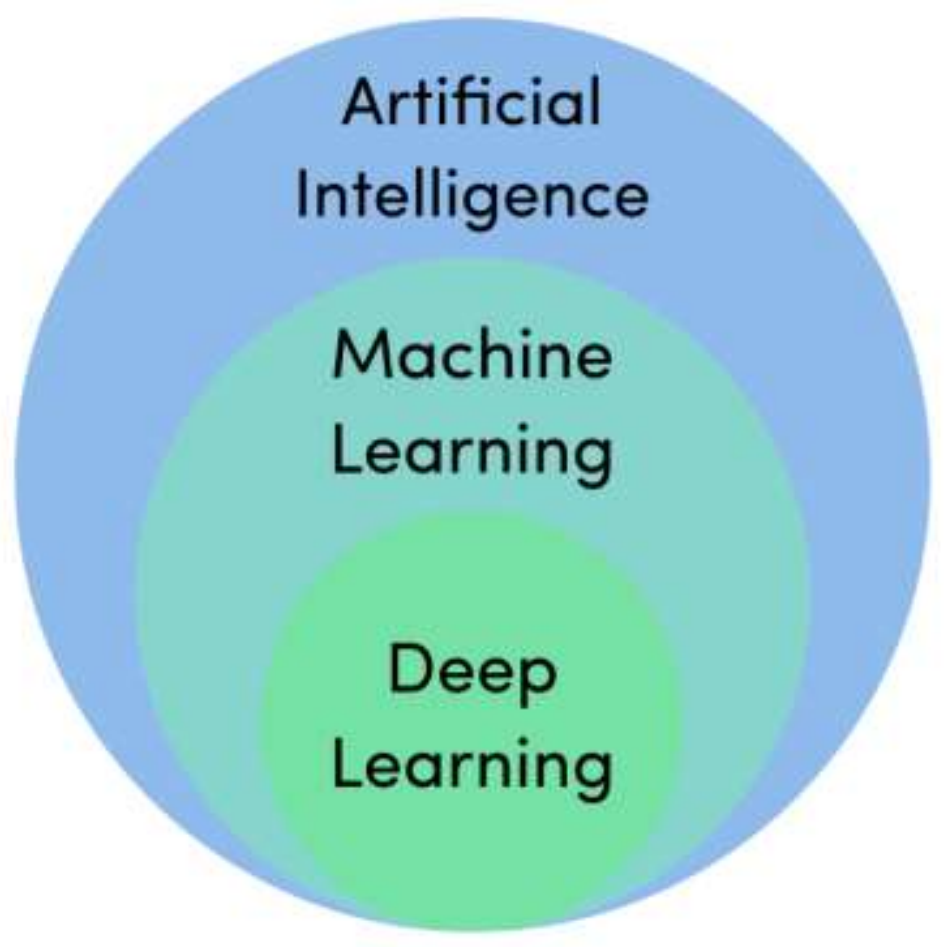

Figure 1. The relationship between AI, ML, and DL [1]. 


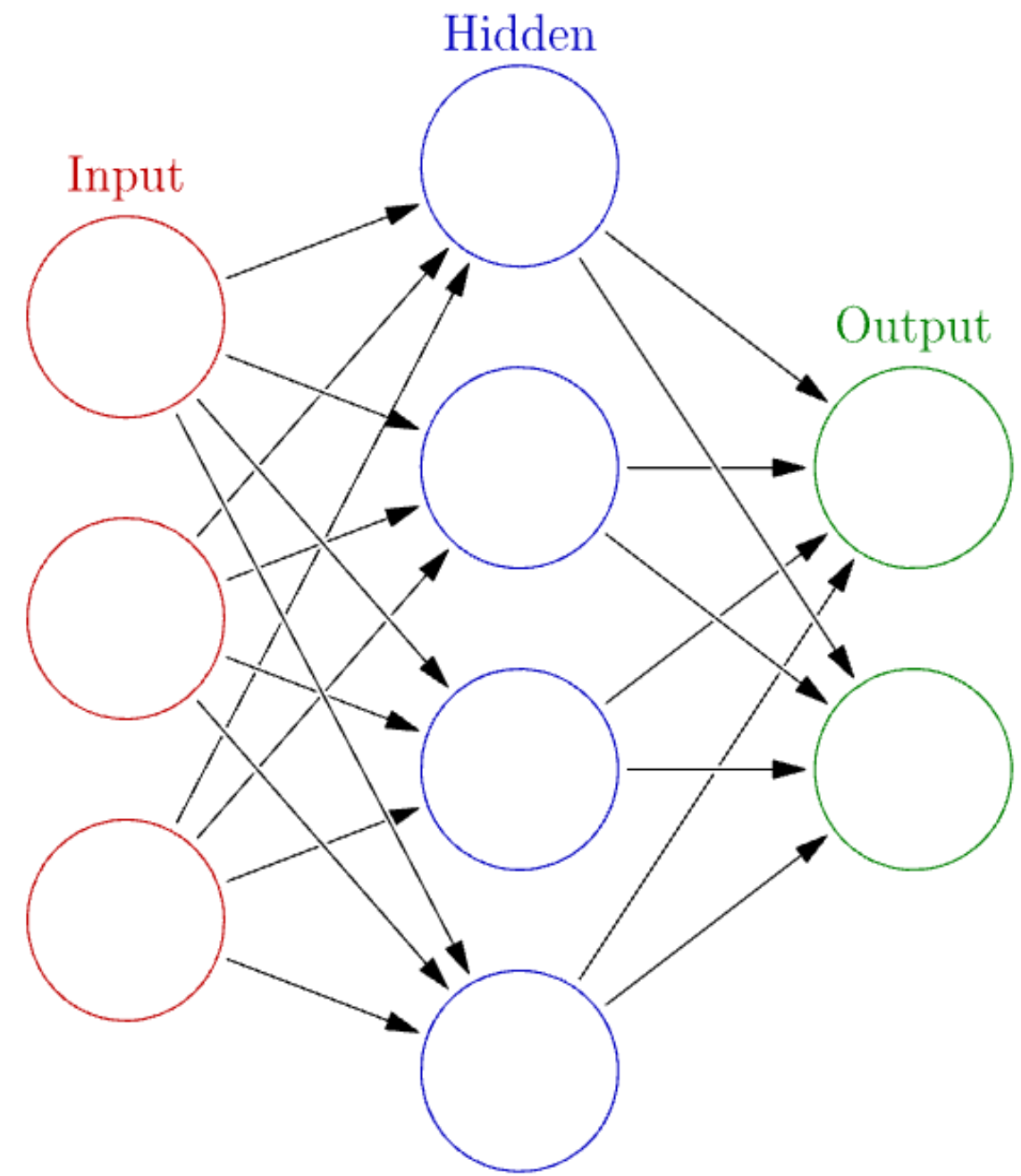

Figure 2. Layers in artificial neural network [4]

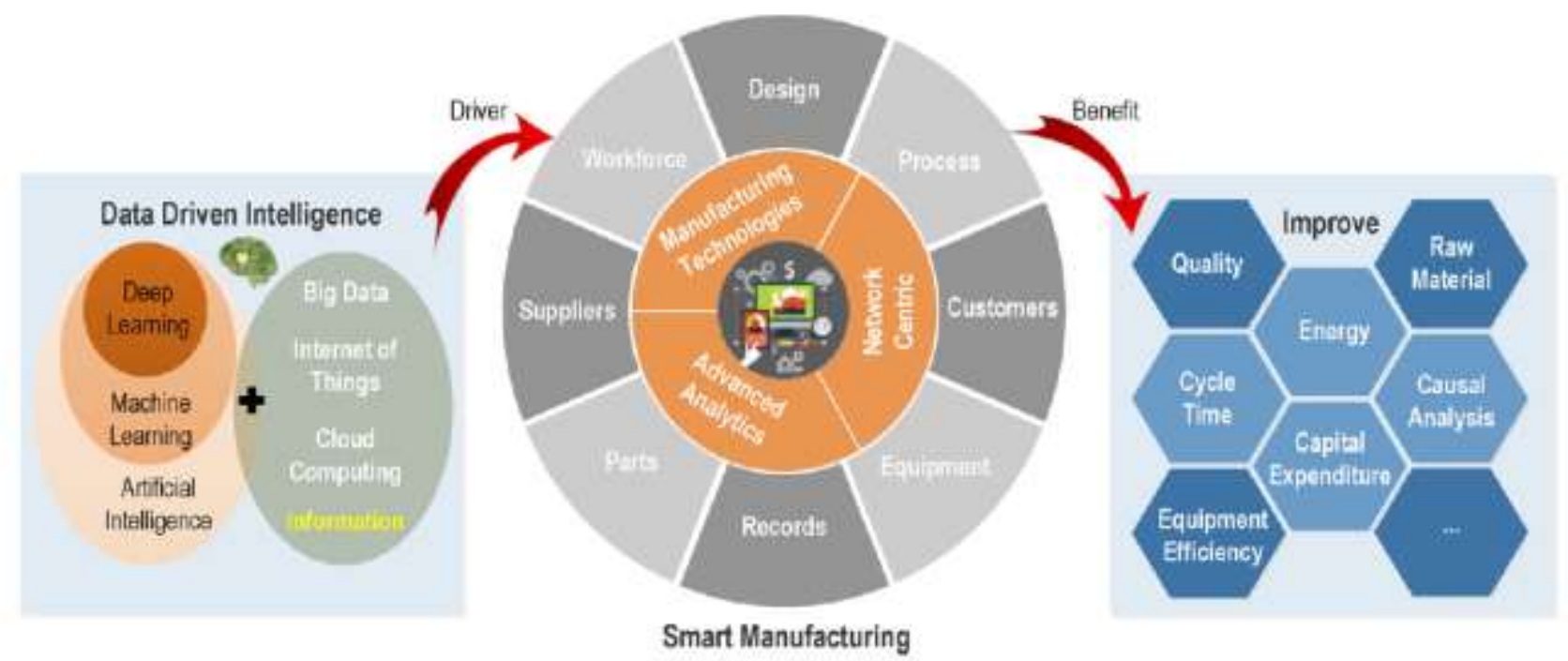

Figure 3. The role of Deep learning in smart manufacturing [7]. 
International Journal of Advances in Scientific Research and Engineering (ijasre), Volume 7 (6), 2021

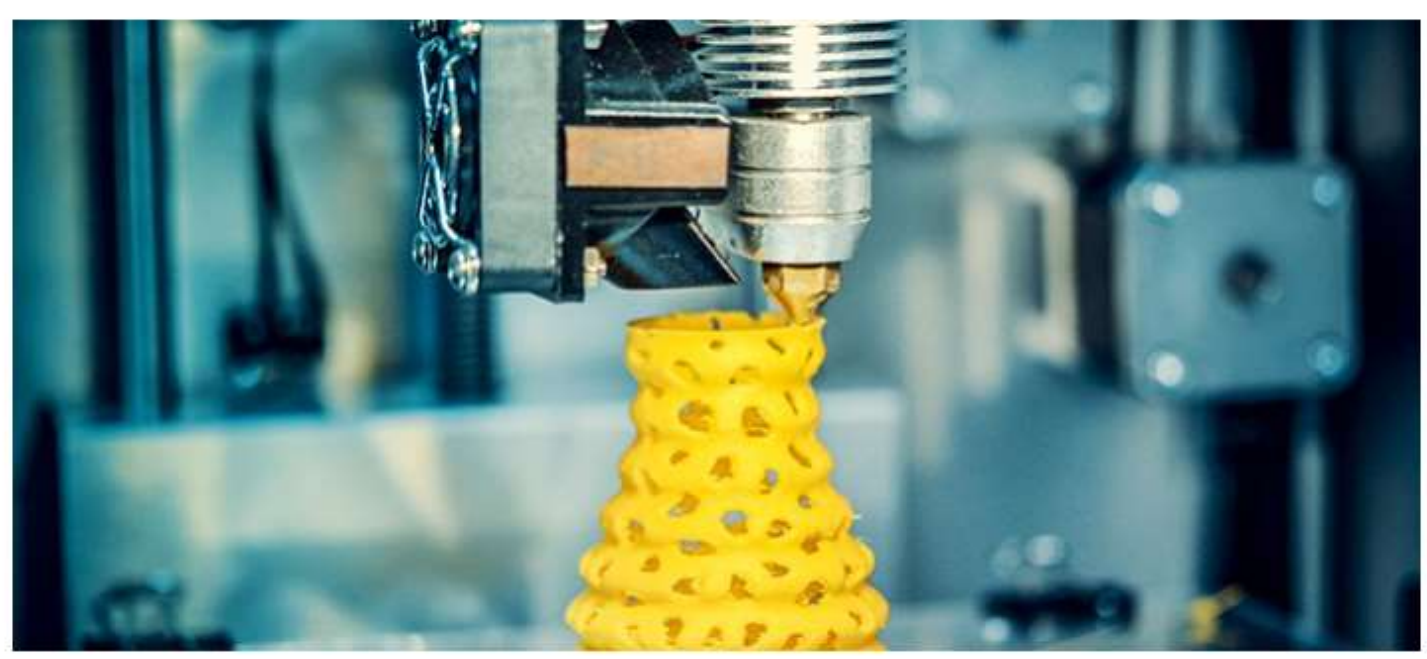

Figure 4. Additive manufacturing [10] 